\title{
A hybrid metaheuristic optimisation technique for load frequency control
}

\author{
Rajeshree Ramjug-Ballgobin ${ }^{1}$ (D) Chiranjeev Ramlukon ${ }^{1}$
}

Received: 18 August 2020 / Accepted: 9 March 2021 / Published online: 12 April 2021

(C) The Author(s) 2021 OPEN

\begin{abstract}
This paper investigates the problem of load frequency control by the application of metaheuristic optimisation techniques. In order to analyse the effect of the system model on the performance of the algorithm, two different two-area systems are considered, modelled using the Matlab/Simulink package. The first one consists of a reheat thermal area and a hydro area while the second one is made up of identical reheat thermal areas. To take into consideration the effects of practical constraints, nonlinearities such as Generation Rate Constraint, Governor Deadband and Boiler Dynamics are introduced. The Shuffled Frog Leaping Algorithm and Teaching Learning Based Optimisation are applied followed by a proposed hybrid of both algorithms to tune Proportional-Integral-Derivative (PID) controllers for the different areas of the systems, taking into account step load changes as inputs. The aim of this proposed algorithm is to merge the qualities of the individual algorithms to provide a more efficient one, converging faster to the optimal gains of the controllers. The results obtained proved the satisfactory performances of all algorithms and superiority of the hybrid Shuffled Frog Leaping Algorithm -Teaching Learning Based Optimisation technique in controlling frequency level in both systems investigated, where the main control measures such as peak values, settling times and steady-state values have been considered.
\end{abstract}

\section{Article Highlights}

- When considering a system of electrical mechanisms installed to produce, distribute and use electric energy, it is important to match supply with demand in order to keep frequency almost constant so as to ensure the safe and reliable use of equipment and maintain stability.
- The combination of two different algorithms efficiently improved the performance of the control system since the new system benefitted from the qualities of both strategies.

- Algorithm-based controllers provided robust and reliable frequency control and may be used to increase the quality of electrical energy under increasing and constantly changing demand.

Keywords Proportional-Integral-Derivative controllers · Shuffled Frog Leaping Algorithm · Teaching Learning Based Optimisation · Load Frequency Control · Generation Rate Constraint · Governor Deadband · Boiler Dynamics

Rajeshree Ramjug-Ballgobin, r.ramjug@uom.ac.mu | 'Electrical and Electronic Engineering Department, Faculty of Engineering, University of Mauritius, Reduit, Mauritius. 


\section{Introduction}

Load Frequency Control (LFC), also termed as power system frequency control or regulation is one of the most important functions of the Automatic Generation Control (AGC). Transient disturbances cause instabilities in large interconnected power system networks [1]. These instabilities affect the normal functioning of the power system by causing discrepancies between load and generation. Automatic frequency and voltage regulators (AVR) are of vital significance at generating stations as the load is never constant in a power system network [2]. With a view to sustain the changing loads, the regulators should be able to contain the frequency and voltage deviations within given limits. The system frequency will shift from the scheduled value if there is a discrepancy between demand and generation and high frequency deviation may cause the system to collapse [3]. The main objective of LFC is to maintain system frequency and tie-line flows between interconnected systems closer to scheduled values as much as possible [2].

Impacts of physical constraints such as dead band, time delays, generation rate and other uncertainties on frequency control in electrical system have been highlighted in the study. Much emphasis has been laid upon the consideration of the important in-built requirements and the basic restrictions introduced by the physical system dynamics in order to get an insightful review of the frequency subject for performance evaluation of reallife systems [4-6]. The most common non-linearities introduced in LFC systems are usually Generation Rate Constraint (GRC), Governor Deadband (GDB) and Boiler Dynamics (BD) for realistic analysis of systems as the incorporated non-linearities have an effect on the settling times and peak values of systems [7].

There has been major development in advanced controllers to ameliorate the transient response of load frequency and to ameliorate the control quality of power systems in spite of the presences of load perturbations and system instabilities [8]. The advanced controllers reported in literature include robust control $[9,10]$, artificial intelligent control [11, 12], adaptive control [13], Model Predictive Control [14], Active Disturbance Rejection Control (ADRC) [15]. In recent years, most works published in the field of frequency control pertain to the use of optimisation techniques to tune controllers, including PID controllers which are mostly used due to simplicity of design [16].

In [17], optimisation techniques such as Genetic Algorithm (GA), Cultural Algorithm (CA) and Jaya-based Invasive Weed Optimization (JIWO), have been utilized to tune PID controllers and the results reported have proved to be satisfactory in improving the transient and steady-state responses of the frequency and tie-line power deviations of the system considered, with JIWO being the better technique in terms of optimality and convergence rate.

An objective function is used for the optimisation of the PID gains controller by evaluating the fitness values of the solutions computed by the objective function for a problem formulated. Some common indices used for the objective function are Integral of Absolute Error (IAE), Integral of Squared Error (ISE), Integral of Time Absolute Error (ITAE) and Integral of Time Squared Error (ITSE) [18]. The ITAE index is normally considered over the other indices for LFC optimisation problems due to its ability to reduce settling times as well as eliminating long duration errors $[19,20]$.

This paper aims to apply the SFLA, TLBO and a proposed hybrid SFLA-TLBO optimisation techniques to optimize the gains of PID controllers for the load frequency control of a two-area system comprising of a reheat thermal and hydro areas and a non-linear two-area system consisting of two identical reheat thermal areas with non-linearities namely GDB, GRC and BD by minimizing the fitness values obtained from the objective function. The performance analysis of the compensated systems in comparison with their uncontrolled counterparts was performed in terms of transient and steady state parameters which are settling times, peak values and the steady-state errors. Also, the optimisation techniques were assessed with respect to the convergence and optimality of the solutions achieved for each system considered. The novelty of the proposed method lies in the combination of two different techniques to extract the benefits of both and hence provide an algorithm which performs better than the individual algorithms.

In this paper, we first consider the models of linear and nonlinear two-area systems and analyze their dynamics (see Sect. 2). Then, two existing optimization techniques are described and the benefit of merging these techniques into hybrid one is investigated (see Sect. 3). All results are presented and discussed in Sect. 4 which is followed by concluding comments in Sect. 5.

\section{System models}

\subsection{Two-area system}

The first system to be considered is a two-area system that comprises of two $2000 \mathrm{MW}, 50 \mathrm{~Hz}$ control areas, one of which is a reheat thermal one while the other one is a hydro one, proposed in [21] without the non-linearities. The model is built on Simulink as presented in Fig. 1. 


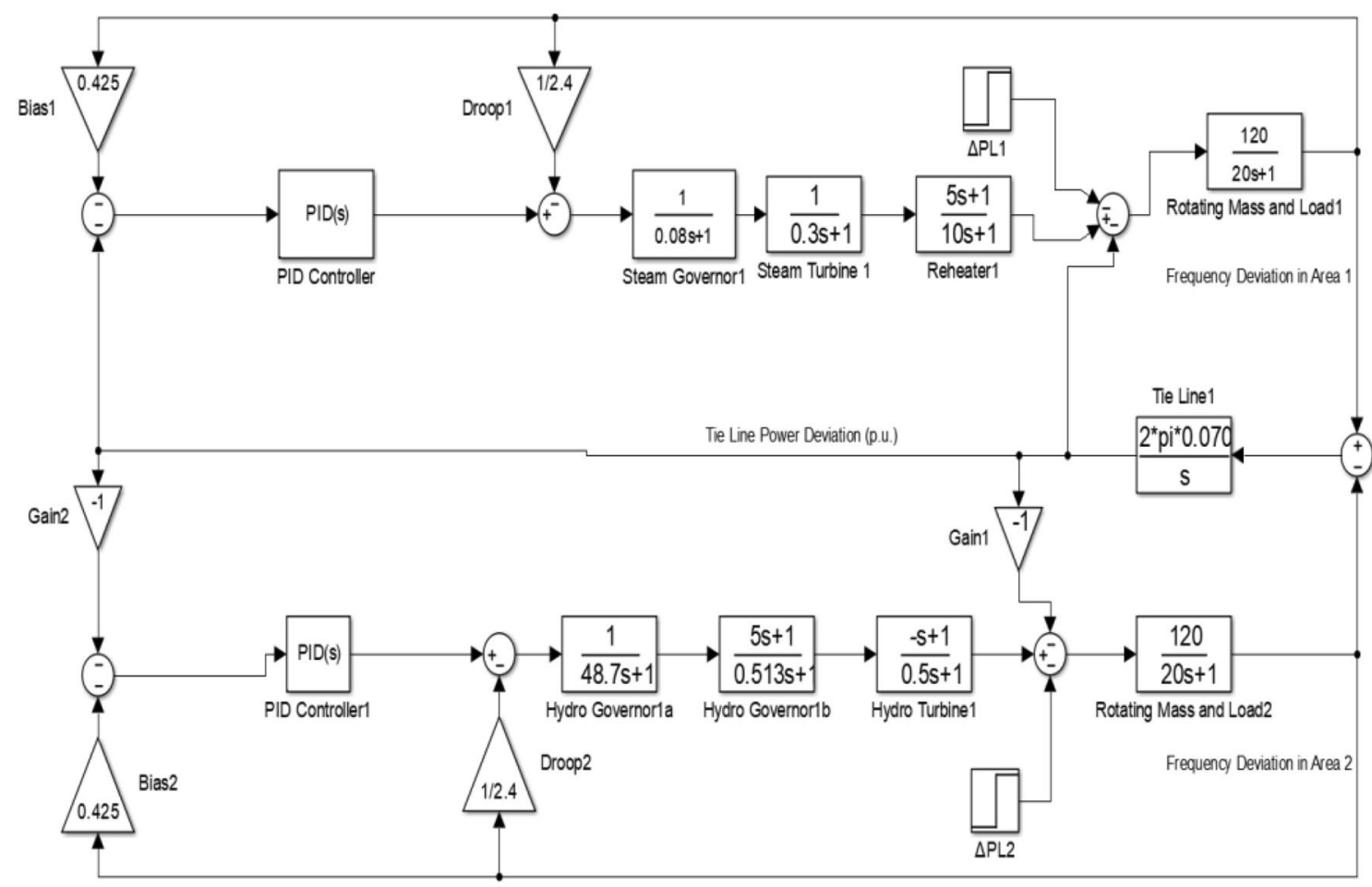

Fig. 1 Linear two-area system

\subsection{Non-linear two-area system}

\subsubsection{Generation rate constraint (GRC)}

Due to mechanical and thermodynamics restrictions in real steam turbines, there are limitations to the rate at which the output power is allowed to fluctuate. Process variables like temperature and pressure are imposed rate limits to avoid large deviations for safety of equipment [7]. Generation rate limitation of $10 \%$ per minute is normally taken into account and is illustrated as by a limiter with \pm 0.0017 p.u. MW/s as bounds.

\subsubsection{Governor deadband (GDB)}

This non-linearity deals with the governor sustaining to speed changes for which the valve's position remains unchanged. For analysis in this study, backlash is chosen to be $0.05 \%$ approximately. A linearised form of the steam governor with GDB is given in Eq. (1) [20]

$G_{\text {sgi }}(s)=\frac{0.8-\frac{0.2}{\pi} s}{1+T_{\text {sgi }} s}$

where $T_{\text {sgi }}$ is the modified governor time constant.

\subsubsection{Boiler dynamics (BD)}

Changes in turbine control valves lead to changes in generations. The required control action is provided by the boiler controls which cause changes in rate of combustion, steam flow, throttle pressure and hence in the boiler output. [20]

The model, basically a drum type boiler, as demonstrated in Fig. 2 shows the representation of the boiler dynamics.

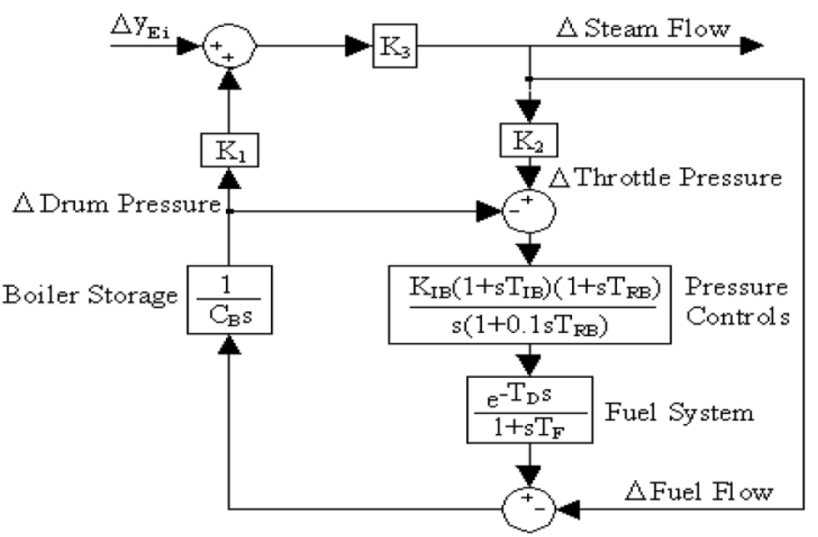

Fig. 2 Boiler dynamics model [20] 
The second system is a two-area system incorporated with non-linearities is based on the model presented in [7]. The proposed Simulink model of the system is presented in Fig. 3.

\section{Optimisation techniques}

\subsection{Objective function}

The ITAE index is used as basis for the objective function for reducing the Area Control Errors (ACEs) of each area for the considered systems.

The ACE for area $i$ is obtained using Eq. (2) [3].

$$
A C E_{i}=\Delta P_{t i e, i}+\beta_{i} \Delta f_{i}
$$

where $\beta_{i}$ is the bias factor.

The objective function is expressed as shown in Eq. (3).

$J=\int_{0}^{t s s}\left(\sum_{\substack{i=1 \\ j=1}}\left|A C E_{i j}\right|\right) d t$

where $i$ is the loading condition, $j$ is the area considered and $t_{s s}$ is the simulation time.
The in-built Simulink PID controller has transfer function as defined by Eq. (4):

$G_{c}(s)=K_{p}+K_{i} \frac{1}{s}+K_{d} \frac{N}{1+N \frac{1}{s}}$

where.

$K_{p}$ is the proportional gain,

$K_{i}$ is the integral gain,

$K_{d}$ is the derivative gain and

$N$ is the filter coefficient, which is set to 100 .

Six variables, which are $K_{p 1}, K_{i 1}$ and $K_{d 1}$, which are the gains for the reheat thermal area controller, and $K_{p 2}, K_{i 2}$ and $K_{d 2}$ for the hydro area controller, are to be optimised by an objective function for the two-area system and for the non-linear system since both areas are identical, only three variables being $K_{p}, K_{i}$ and $K_{d}$ are required.

The loading conditions for the two-area system are given as Step Load Perturbations (SLP) as shown in Table 1.

The loading conditions for the non-linear two areas are given in Table 2 .

The optimisation process is formulated as follows:

Minimize J

Subject to

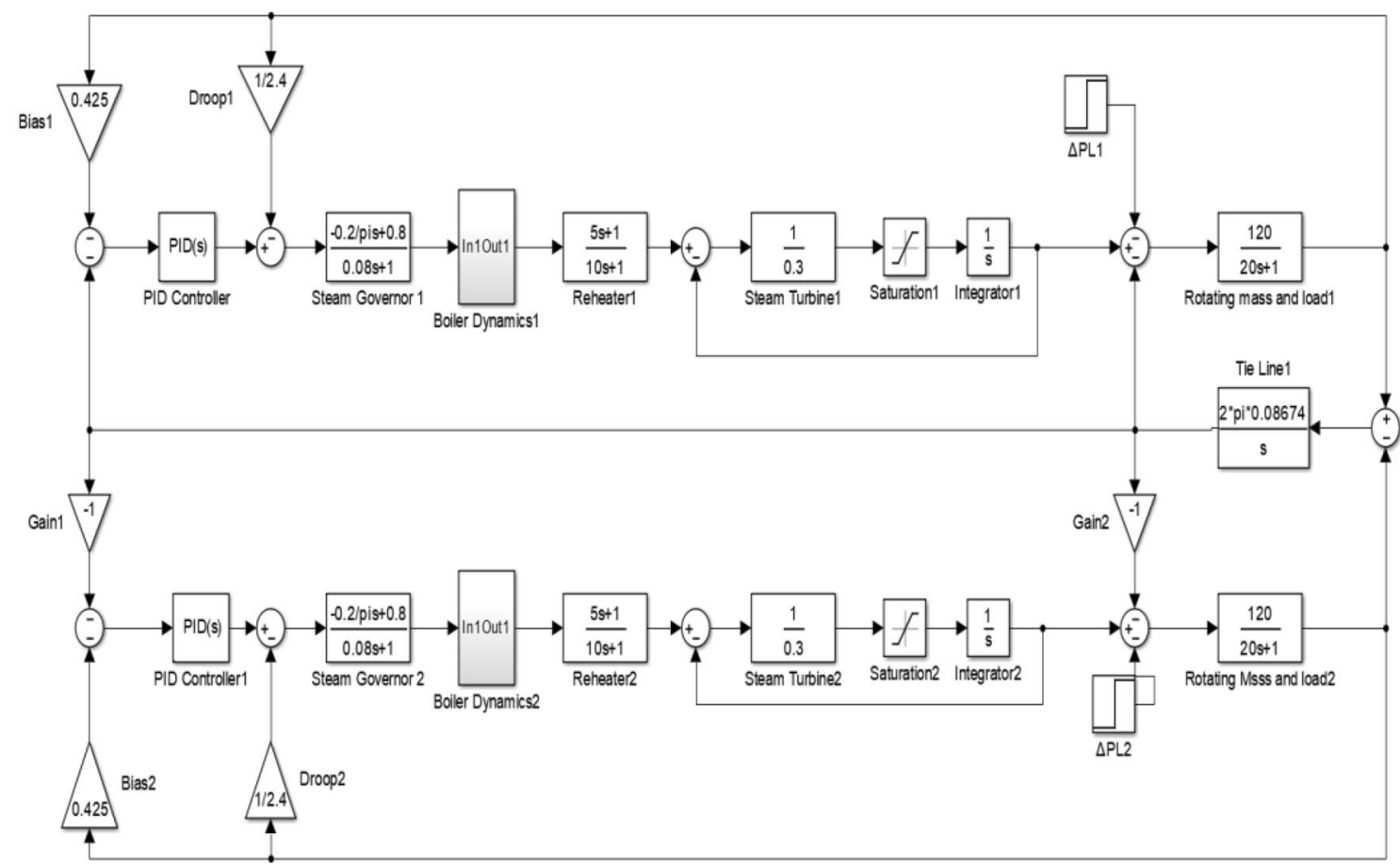

Fig. 3 Non-linear two-area system 


$$
\left\{\begin{aligned}
K_{p \min } & \leq K_{p} \leq K_{p \max } \\
K_{i \min } & \leq K_{i} \leq K_{i \max } \\
K_{d \text { min }} & \leq K_{d} \leq K_{d \max }
\end{aligned}\right.
$$

where the lower and upper bounds of the controller gains are chosen as 0 and 2 , respectively. [16, 23]

\subsection{Shuffled leaping frog algorithm (SFLA)}

The SFLA is a recently popular meta-heuristic algorithm proposed in 2003 by Eusuff and Lansey [24]. It is based on the memetic evolution concept which is a populationbased methodology to solve optimisation problems heuristically and the concept has been recognized widely for its ability to solve large combinatorial optimisation problems which other meta-heuristics could not [25]. Numerous parallel communities, known as memeplexes are partitioned within the population and they are allowed to evolve autonomously so as to explore the search space in different directions [26].

The total population size, denoted by $\mathrm{F}$ is calculated by Eq. (5).

$F=m \times n$

where $m$ represents the number of memeplexes and $n$ the number of frogs in each memeplex.

Within each memeplex, the frogs are inspired by the ideas of their counterparts and hence they undergo memetic evolution through a process named as local search whose aim is to ameliorate the quality by enhancing the performance of each individual frog towards the optimal solution [27]. During local search, submemeplexes are created by firstly sorting all the frogs in each memeplex according to decreasing fitness values as evaluated by the objective function for each frog and then a triangular probability function is used to select the frogs based on the ranking in the ordered list. The frog having the best fitness value is identified as $X_{b}$ and the one with the worst fitness value as $X_{w}$ within each memeplex. $X_{g}$ represents the solution with the global best fitness value.

The position of the worst frog is modified as given by Eq. (6):

$X_{w(\text { new })}=X_{w}+S_{i}$

where $S_{i}$ being the change in position is given by Eq. (7):

$S_{i}=$ rand $\times\left(X_{b}-X_{w}\right)$

$-S_{\max } \leq S_{i} \leq S_{\max }$

where $S_{\max }$ denotes the maximum step size equal to the number of decision variables in the optimisation problem which the infected frog is allowed to adopt and rand is a random number in the range $[0,1]$.

If this procedure does not generate an improved solution, the calculation in Eq. (6) is repeated by considering the global best frog in the population in which $X_{b}$ is replaced by $X_{g}$. If the solution is still not improved, a new frog (solution) is generated randomly to substitute the worst frog whose position was adverse to improvement. The memeplexes are then forced to blend with other ones through a shuffling procedure, forming new memeplexes. This shuffling process enriches the quality of the frogs after being infected by the ones belonging to different sections for a number of iterations. [24]

The main parameter settings for the implementation of the SFLA are as follows:

- Memeplex size $=10$

- Number of memeplexes $=10$

- Number of submemeplexes $=3$

- Maximum number of iterations for local search $=5$

- Maximum Step size $=2$

The total population size as calculated by Eq. (5) is 100 and the stopping criterion is given by the maximum number of iterations which is set to 100 for both systems.

\subsection{Teaching learning based optimisation (TLBO)}

Teaching Learning Based Optimisation is a relatively recent developed algorithm devised by Rao et al. [28] emulating the roles of a teacher and students in a classroom. There are two main modes of learning considered in this technique which are through the teacher, referred to as the teacher phase and the learner phase where the learners interact with his/her peers [29]. TLBO is mostly recognized for its non-requirement of specific algorithm variables [30].

\subsubsection{Teacher phase}

This phase begins by defining the best solution of the population being the teacher as the latter owns a higher knowledge level than his/her learners. The main goal of the teacher is to enhance the knowledge of the learners. The teacher attempts to improve the mean knowledge level to a better mean value obtained by conducting a survey of knowledge levels in the class to determine each learner's level [31].

This aspect is presented mathematically as follows in which $X_{j, k, i}$ represents any solution in a population assumed to be a group of $n$ learners and $m$ is designated to be the different design variables which represent the different subjects proposed to the students in the optimisation space where 
- $j$ is the subject taken by the students, meaning the $j$ th design variable where $j=1,2, \ldots, m$;

- $k$ is a leaner, representing the $k$ th population member where, $k=1,2, \ldots n$

- $i$ representing the ith iteration, $i=1,2, \ldots, I_{\max }$, where $I_{\max }$ is the maximum number of iterations.

$X_{k, i}$ is denoted as the best solution at any iteration $i$ for which its function value is minimum among the population.

The mean grade of the students in specific subject $j$ is represented as $M_{j, i}$. Equation (8) gives the improvement in the original mean result in each subject by the teacher as follows:

Diff_Mean $n_{j, k, i}=r_{j, i}\left(X_{j, k b e s t, i}-T_{F} M_{j, i}\right)$

where $X_{j, k b e s t, i}$ is the grade of the teacher in subject $j, T_{F}$ denotes the teaching factor (capacity of the teacher) which decides on whether to alter the value of mean having a value of 1 or 2 and $r_{j, i}$ is the random number in the range $[0,1]$.

The existing solution is adjusted in accordance to the Diff_Mean ${ }_{j, k, i}$ in the teacher phase according to Eq. (9):

$X_{j, k, i}^{\prime}=X_{j, k, i}+$ Diff_Mean ${ }_{j, k, i}$

where $X_{j, k, i}^{\prime}$ denotes the updated $X_{j, k, i}$ value.

If the computed value of $X_{j, k, i}^{\prime}$ results into a better function value than $X_{j, k, i}$, the former is retained, discarding the latter. The best function values are retained after completing the teacher phase and used as inputs to the learner's phase.

\subsubsection{Learner phase}

In this phase, a learner increases his knowledge level if the other learners have better knowledge than he does. At any iteration $i$, there is comparison between one learner and his peers randomly. Similar to the teacher phase, the best function values obtained and accepted at completion of this phase are retained and used as inputs for the next iteration's teacher phase.

For the parameter settings of the TLBO, only the population size and the stopping criterion being the maximum number of iterations have to be set prior to simulating the system models as TLBO is a parameter-free algorithm. Both the population size and maximum iterations number are taken to be 100 .

\subsection{Hybrid SFLA-TLBO}

This proposed algorithm is a combination of the SFLA and TLBO methods employed in cascade form. The objective of this algorithm is to use the properties of both techniques as a means to enhance the search efficiency. In this approach, the fitness values generated by both algorithms are compared and the best fitness value and position from either algorithm is retained for the succeeding iteration.

If the new computed fitness value computed by TLBO is better than the SFLA's one, the improved one is retained, otherwise the new fitness value is rejected, retaining the SFLA's one for inputs for the next iteration. The same individual parameter settings as laid out in Sect. 3.2 and 3.3 for SFLA and TLBO respectively are used for this hybrid technique.

Figure 4 demonstrates the flowchart explaining the hybridization and implementation process of the algorithm.

\section{Results and discussions}

\subsection{Two-area system}

The optimal PID gains obtained by the optimisation techniques are tabulated as in Table 3 along with the corresponding best fitness values.

Figure 5 shows convergence profiles of the optimisation techniques towards the best solutions and Fig. 6 is a magnified view of the rates of the convergence for each technique.

All four optimisation techniques employed for tuning the gains of the PID controllers by optimizing the objective functions produce practically the same results as the best fitness value are almost the same, leading to virtually the same PID gains. This is primarily due to the limits constraints imposed on the PID gains which are 0 as minimum limit and 2 as maximum limit, and also the large number of iterations ran during the optimisation process.

The slight difference in fitness value for SFLA is mainly due to parameter settings for instance the maximum step size and number of memetic evolutions in the local search process among others. Capitalizing on the properties of TLBO, SFLA has been modified to create a proposed hybrid algorithm, SFLA-TLBO, where the algorithms have been implemented in a sequential manner. Consequently, the proposed SFLA-TLBO proves to be the most superior optimisation technique, taking only around 15 iterations to find the optimal solution.

Figures 7, 8 and 9 show the responses of the system subject to step load changes of 0.01 p.u. in Area 1 . 
Fig. 4 Hybrid SFLA-TLBO flowchart

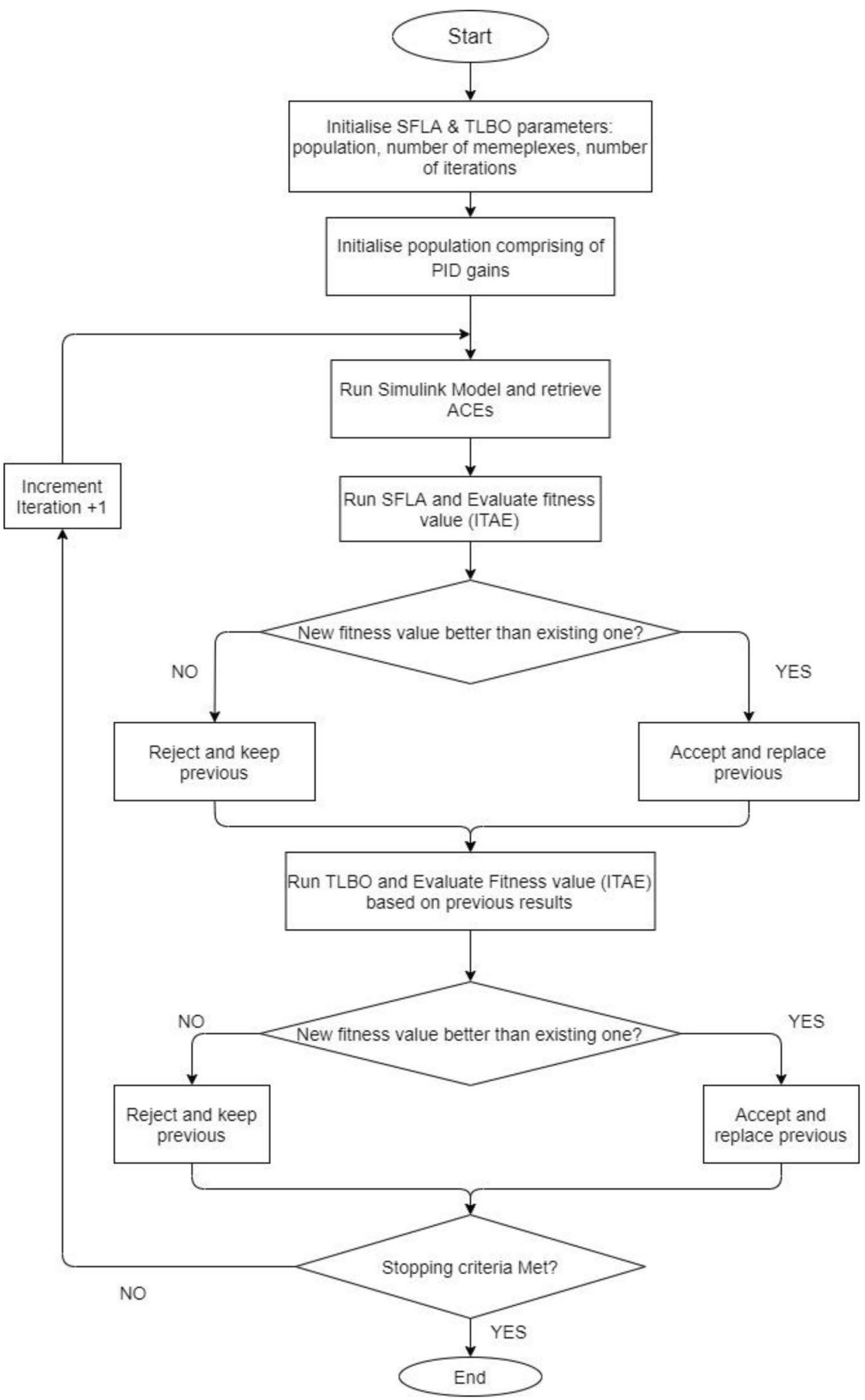

Table 4 provides a breakdown of the performance measures of the controlled and uncontrolled systems.

The settling times have been reduced by $77.3 \%$ for frequency deviations in Area 1, 82.5\% for Area 2 and $76.6 \%$ for tie-line power deviation and the peak values are improved by $70.0 \%$ and $59.4 \%$ for frequency deviation in Area 1 and Area 2 respectively, and $55.7 \%$ for tie-line 
Table 1 Loading conditions for linear two-area system

\begin{tabular}{lll}
\hline Loading conditions & Area 1 SLP (p.u.) & Area 2 SLP (p.u.) \\
\hline 1 & 0.01 & 0 \\
2 & 0 & 0.01 \\
3 & 0.01 & 0.01 \\
4 & 0.02 & 0 \\
5 & 0 & 0.02 \\
\hline
\end{tabular}

Table 2 Loading conditions for non-linear two-area system

\begin{tabular}{lll}
\hline Loading conditions & Area 1 SLP (p.u.) & Area 2 SLP (p.u.) \\
\hline 1 & 0.01 & 0 \\
2 & 0.01 & 0.01 \\
3 & 0.02 & 0 \\
4 & 0.02 & 0.01 \\
\hline
\end{tabular}

penalised at the expense errors that occur at the very beginning of response. Therefore, the proportional and integral components are mostly at their maximum limits in all the controllers and by virtue of their function, they promote increase in overshoot.

\subsection{Non-linear two-area system}

The optimal fitness values obtained by the optimisation processes are exactly the same, leading to exactly same PID gains. Table 5 shows the optimal PID gains with the corresponding fitness values.

Figure 10 shows convergence profiles of the optimisation techniques towards the best solutions and Fig. 11 is a magnified view of the rates of the convergence for each technique.

All the optimisation techniques achieve optimality at different points in time. TLBO converges faster than SFLA
Table 3 Optimal solutions for two-area system

\begin{tabular}{llllllll}
\hline $\begin{array}{l}\text { Optimisation } \\
\text { techniques }\end{array}$ & Best fitness & \multicolumn{2}{l}{ PID gains } & & & \\
\cline { 2 - 8 } & & $K_{p 1}$ & $K_{i 1}$ & $K_{d 1}$ & $K_{p 2}$ & $K_{i 2}$ & $K_{d 2}$ \\
\hline SFLA & 2.3096 & 1.9998 & 2 & 0.4749 & 1.0690 & 2 & 1.4073 \\
TLBO & 2.3095 & 2 & 2 & 0.4810 & 1.0716 & 2 & 1.4070 \\
SFLA-TLBO & 2.3095 & 2 & 2 & 0.4810 & 1.0716 & 2 & 1.4070 \\
\hline
\end{tabular}

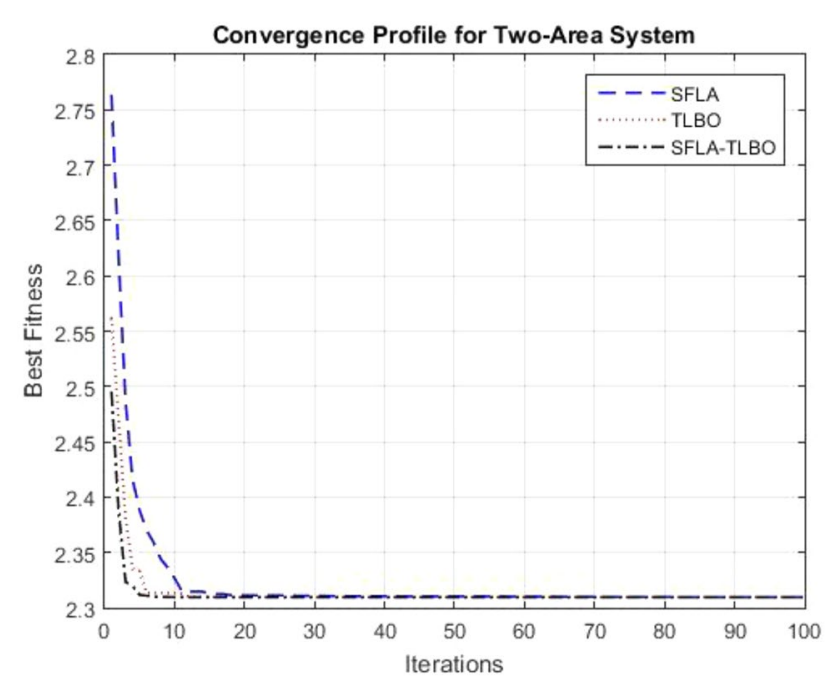

Fig. 5 Convergence profile for two-area system

power deviation. Above all, the steady-state errors have been completely eliminated.

Although the system settles faster with zero steady state error using all the controllers designed, there is occurrence of peak overshoots in frequency deviations. This is due to the use of ITAE index as objective function in which errors that persist for a long duration are

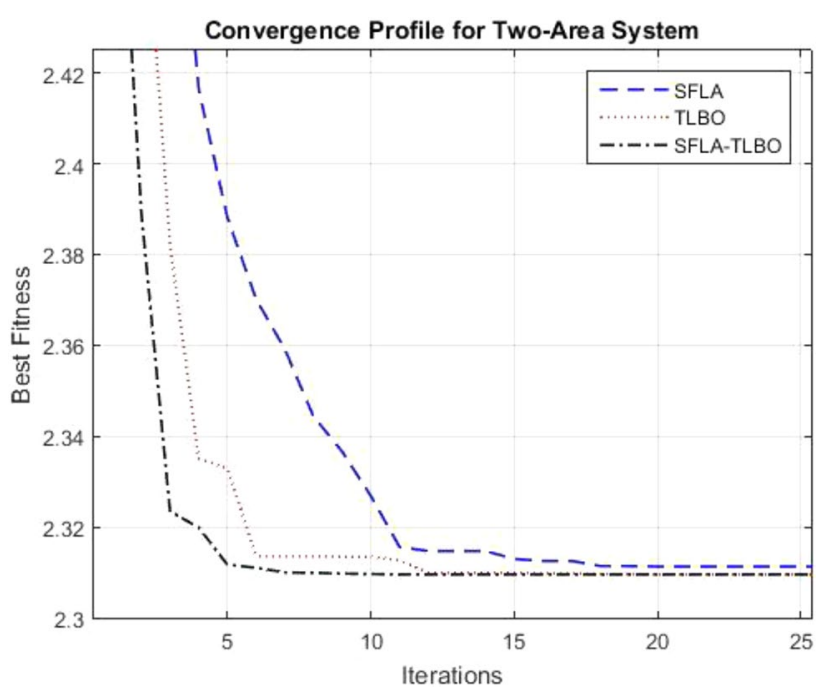

Fig. 6 Convergence profile for two-area system (magnified)

towards the best solution. The proposed SFLA-TLBO, once again produces superior results in terms of optimality and convergence requiring the least number of iterations to find the optimal fitness value. 
Fig. 7 Frequency deviation in area 1 for two-area system

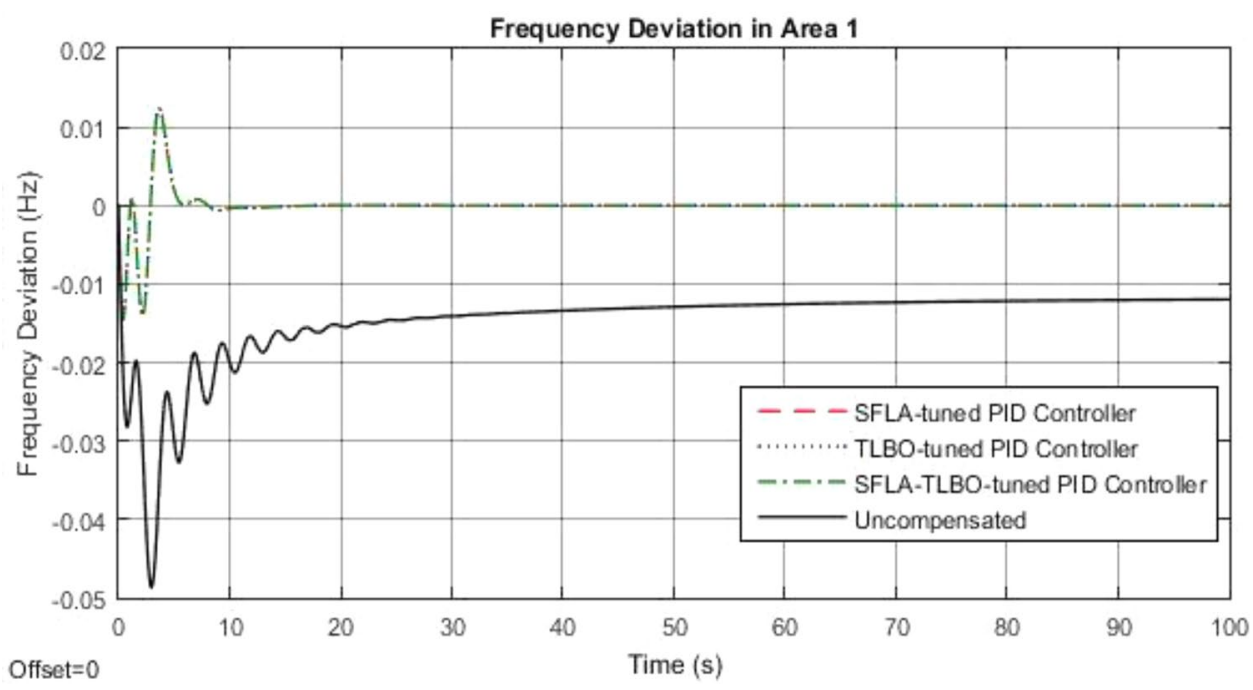

Fig. 8 Frequency deviation in area 2 for two-area system

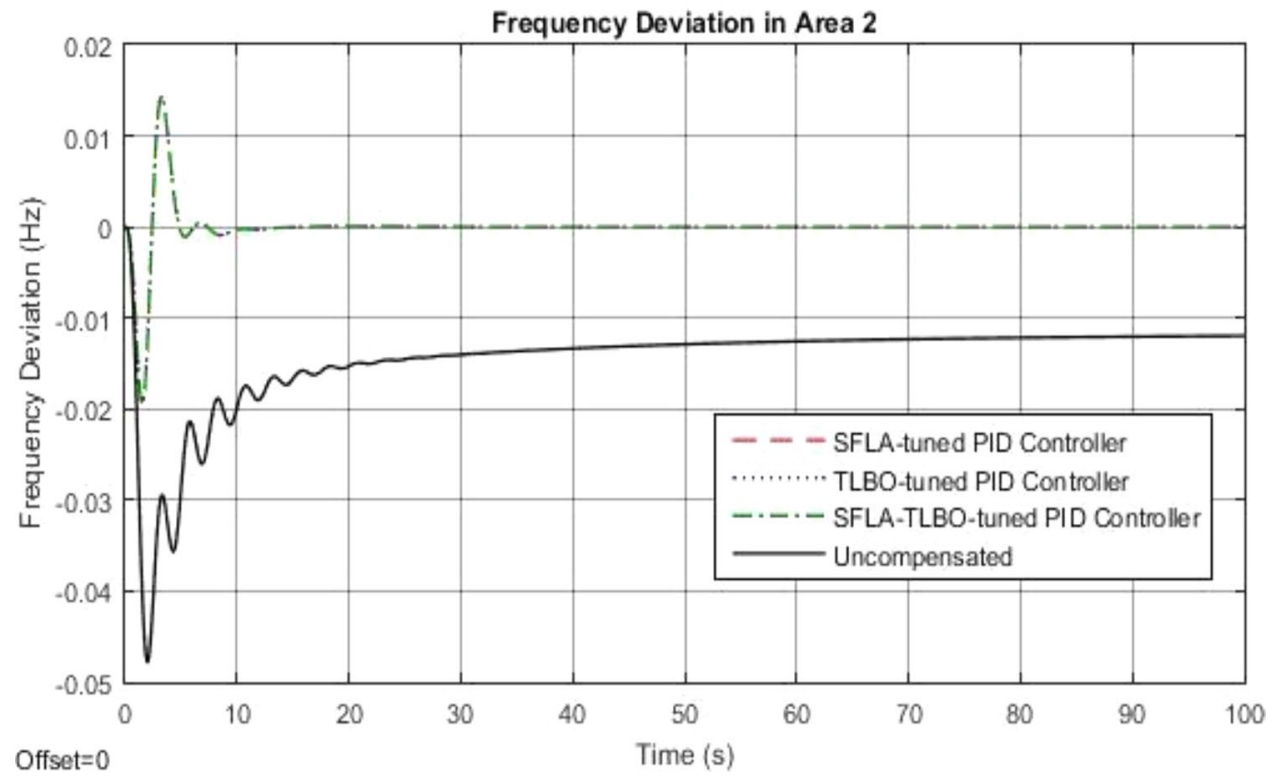

Figures 12,13 and 14 show the responses of the system subject to same loading conditions as for the linear twoarea system.

The performance measures for the compensated and uncompensated systems are compared as shown in Table 6.

Upon effect of the controllers, the frequency deviations settle 59\% faster but for tie-line power deviation, the settling time deteriorates considerably as the value increases by about six-fold the uncompensated value, mainly due to the high derivative gain. There is also an increase of $32 \%$ in the peak values of the frequency deviations, but an improvement of $7.3 \%$ for the tie-line power deviation. All the steady state errors are eliminated by the controllers.

\section{Conclusion}

Three intelligent optimization techniques namely, SFLA, TLBO and a proposed SFLA-TLBO have been used to tune the gains PID controllers for LFC systems. The main findings of this work are as follows:

1. The first system considered is a two-area system consisting of a reheat thermal area and a hydro one. The optimization processes have resulted in similar fitness values, thus identical PID gains. Therefore, the performances of the controllers on the system are very alike. From the responses, it can be deduced that the effects of the controllers on the system are satisfactory as they 
Fig. 9 Tie-line power deviation for two-area system

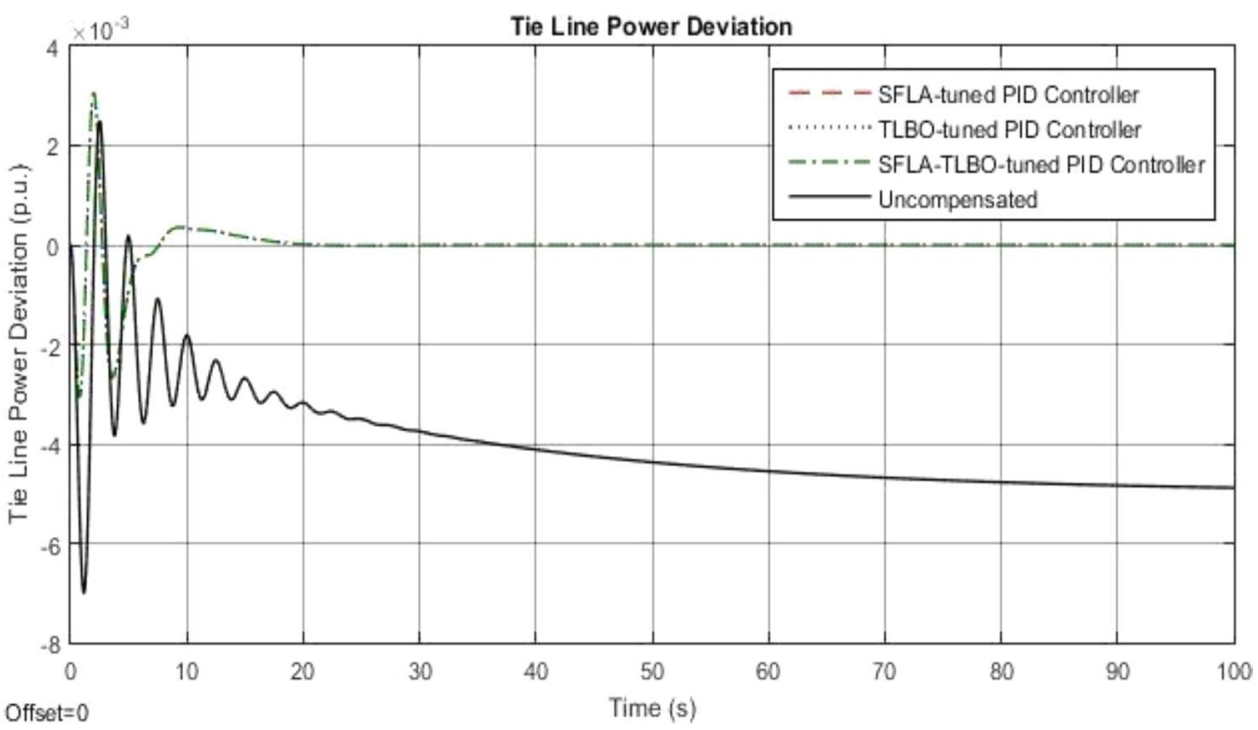

\begin{tabular}{llllll}
\hline Performance measure & Parameter & Uncompensated & SFLA & TLBO & SFLA-TLBO \\
\hline Settling time (s) & $\Delta \mathrm{f}_{1}(\mathrm{~Hz})$ & 56.2983 & 12.8057 & 12.8055 & 12.8055 \\
& $\Delta \mathrm{f}_{2}(\mathrm{~Hz})$ & 55.8856 & 9.7820 & 9.7669 & 9.7669 \\
\multirow{2}{*}{ Peak value } & $\Delta \mathrm{P}_{\text {tie }}$ (p.u.) & 75.6221 & 17.6617 & 17.6715 & 17.6715 \\
& $\Delta \mathrm{f}_{1}(\mathrm{~Hz})$ & -0.0487 & -0.0146 & -0.0146 & -0.0146 \\
& $\Delta \mathrm{f}_{2}(\mathrm{~Hz})$ & -0.0478 & -0.0194 & -0.0194 & -0.0194 \\
Steady state value & $\Delta \mathrm{P}_{\text {tie }}$ (p.u.) & -0.0070 & -0.0031 & -0.0031 & -0.0031 \\
& $\Delta \mathrm{f}_{1}(\mathrm{~Hz})$ & -0.0120 & 0 & 0 & 0 \\
& $\Delta \mathrm{f}_{2}(\mathrm{~Hz})$ & -0.0120 & 0 & 0 & 0 \\
& $\Delta \mathrm{P}_{\text {tie }}$ (p.u.) & -0.0049 & 0 & 0 & 0 \\
\hline
\end{tabular}

Table 4 Performance analysis for two-area system

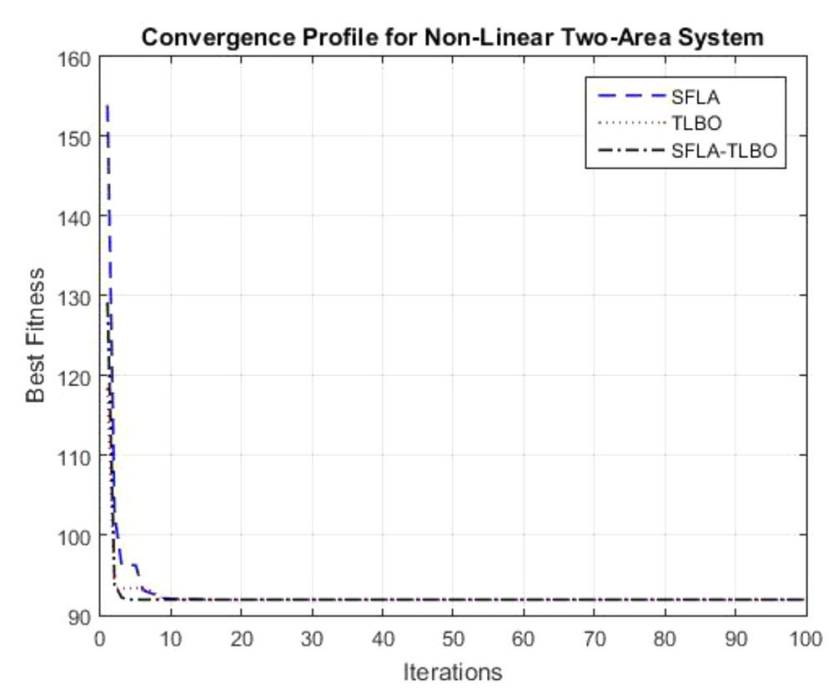

Fig. 10 Convergence profile for non-linear two-area system
Table 5 Optimal solutions for non-linear two-area system

\begin{tabular}{lllll}
\hline \multirow{2}{*}{$\begin{array}{l}\text { Optimisation } \\
\text { techniques }\end{array}$} & Best fitness & \multicolumn{3}{l}{ PID gains } \\
\cline { 3 - 5 } & & $K_{p}$ & $K_{i}$ & $K_{d}$ \\
\hline SFLA & 91.9414 & 0 & 0.0227 & 2 \\
TLBO & 91.9414 & 0 & 0.0227 & 2 \\
SFLA-TLBO & 91.9414 & 0 & 0.0227 & 2 \\
\hline
\end{tabular}

achieve to improve the transient response by reducing the settling times and peak values and at the same time, more essentially, eliminate completely the steady state errors. The controllers have also been tested for robustness against a number of load changes and the results achieved are as desired.

2. The same techniques have been applied to a three area system consisting of two identical reheat thermal areas and a hydro along with a non-linear twoarea system consisting of two identical reheat thermal areas along with generation rate constraints, governor 


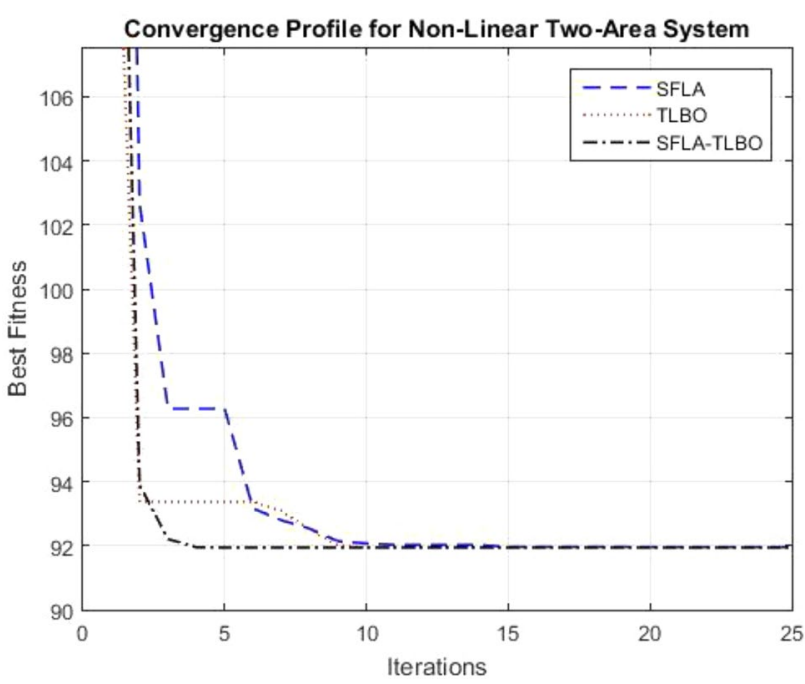

Fig. 11 Convergence profile for non-linear two-area system (magnified)

dead band and boiler dynamics as real-life systems are based on the latter.

3. Similar to the two-area system, the fitness values of the optimization processes for both systems are identical; thereby the gains of the controllers are also similar, resulting in similar performances.

4. For the three-area system, it is observed that the controllers succeed in removing all steady state errors and to a major extent improving the transient responses.
5. The non-linear system is more complex due to the non-linearities present to delay the system to reach synchronism.

6. As for the two previous systems, the steady state errors in the responses are eliminated. The transient responses for the frequency deviation are bettered but not quite for the tie line power deviation as the settling time is increased considerably. Being tested for robustness, the non-linear system demonstrated, once again, satisfactory results in terms of steady state response, however it is noted that the compensated transient responses are dependent on the load changes.

7. The performances of each approach for optimizing the PID gains have also been assessed in terms of convergence rate towards the optimal solution. All the optimization methods achieve optimality but at different points in time. Since TLBO is a parameter-free algorithm, its convergence rate is faster than that of SFLA where some parameters have to be set.

As further work, it is recommended to adapt the proposed technique to other applications such as voltage regulation and also carry out a study about the proper selection of parameter settings for the SFLA technique with the view of improving the optimality and convergence rate of the approaches.
Fig. 12 Frequency deviation in area 1 for non-linear two-area system

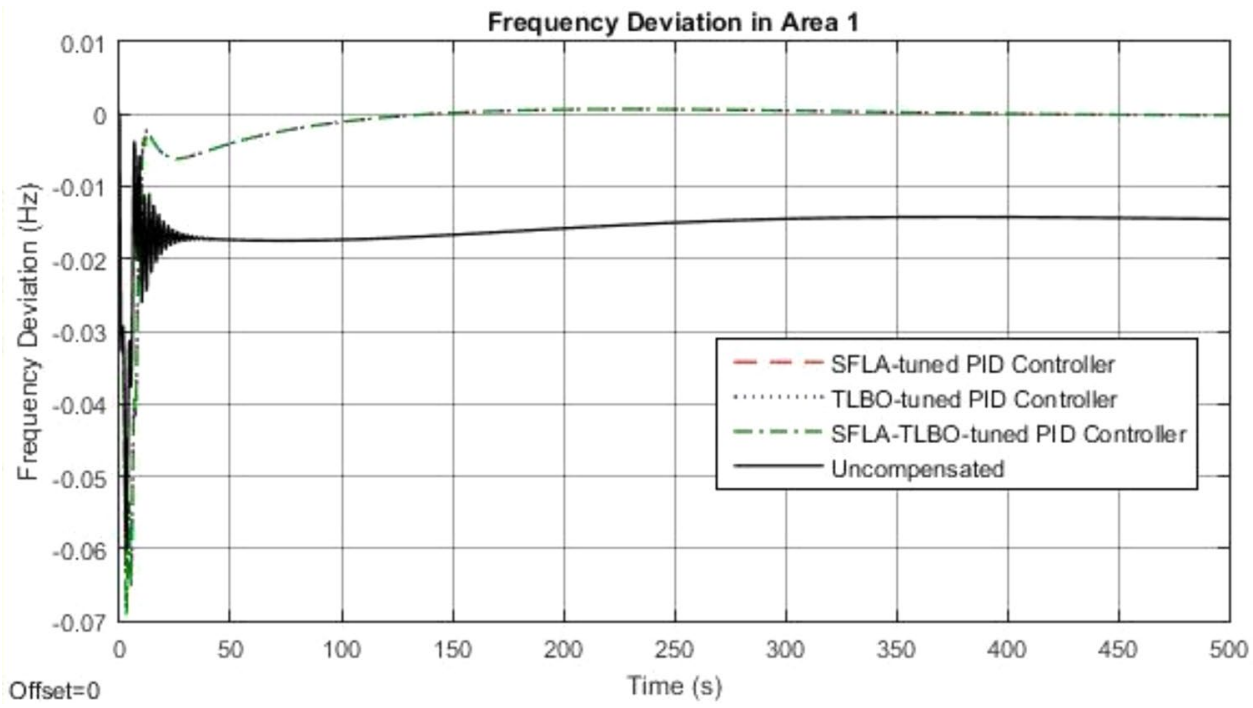

SN Applied Sciences 
Fig. 13 Frequency deviation in area 2 for non-linear two-area system

Table 6 Performance analysis for non-linear two-area system

Fig. 14 Tie line power deviation for non-linear two-area system

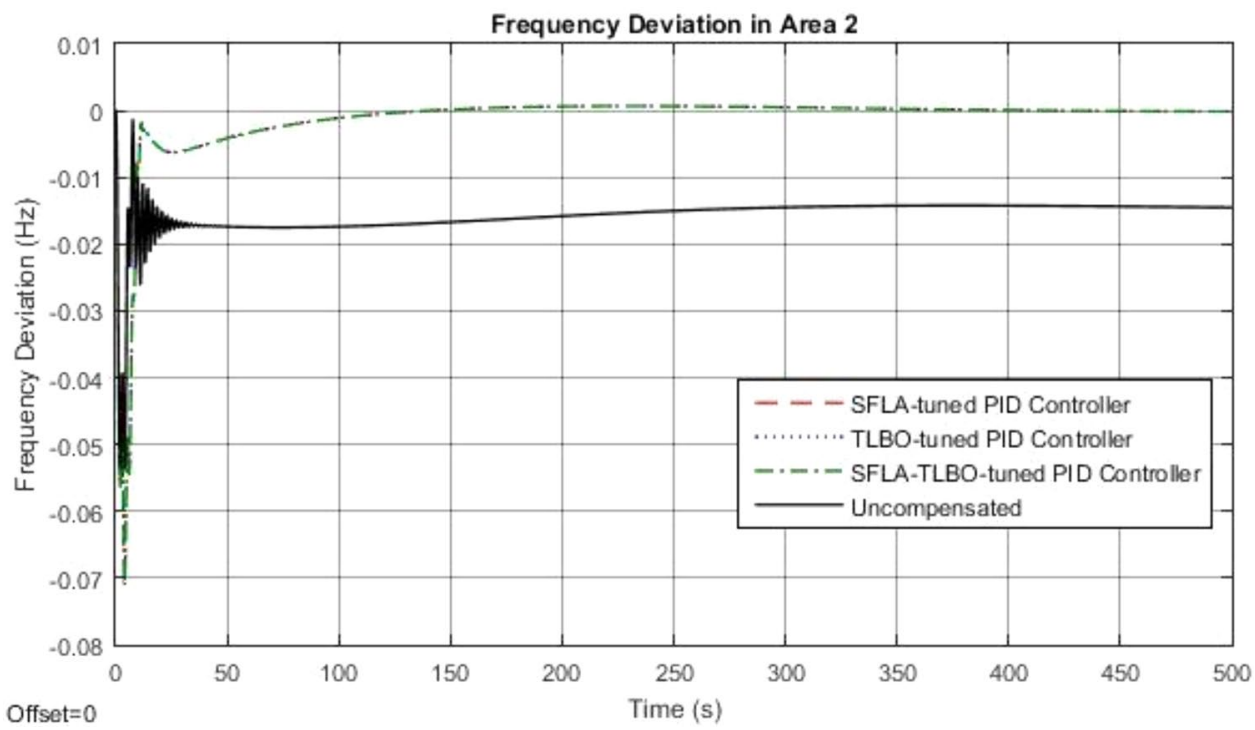

\begin{tabular}{llllll}
\hline Performance measure & Parameter & Uncompensated & SFLA & TLBO & SFLA-TLBO \\
\hline Settling time $(\mathrm{s})$ & $\Delta \mathrm{f}_{1}(\mathrm{~Hz})$ & 210.2266 & 89.4812 & 89.4812 & 89.4812 \\
& $\Delta \mathrm{f}_{2}(\mathrm{~Hz})$ & 218.0827 & 89.5541 & 89.5541 & 89.5541 \\
& $\Delta \mathrm{P}_{\text {tie }}(\mathrm{p}$. u. $)$ & 32.2370 & 190.0832 & 190.0832 & 190.0832 \\
Peak value & $\Delta \mathrm{f}_{1}(\mathrm{~Hz})$ & -0.060354 & -0.069175 & -0.069175 & -0.069175 \\
& $\Delta \mathrm{f}_{2}(\mathrm{~Hz})$ & -0.053718 & -0.071017 & -0.071017 & -0.071017 \\
\multirow{2}{*}{ Steady state value } & $\Delta \mathrm{P}_{\text {tie }}($ p.u. $)$ & -0.009387 & -0.008703 & -0.008703 & -0.008703 \\
& $\Delta \mathrm{f}_{1}(\mathrm{~Hz})$ & -0.014706 & 0 & 0 & 0 \\
& $\Delta \mathrm{f}_{2}(\mathrm{~Hz})$ & -0.014706 & 0 & 0 & 0 \\
& $\Delta \mathrm{P}_{\text {tie }}($ p.u. $)$ & -0.0050 & 0 & 0 & 0 \\
\hline
\end{tabular}

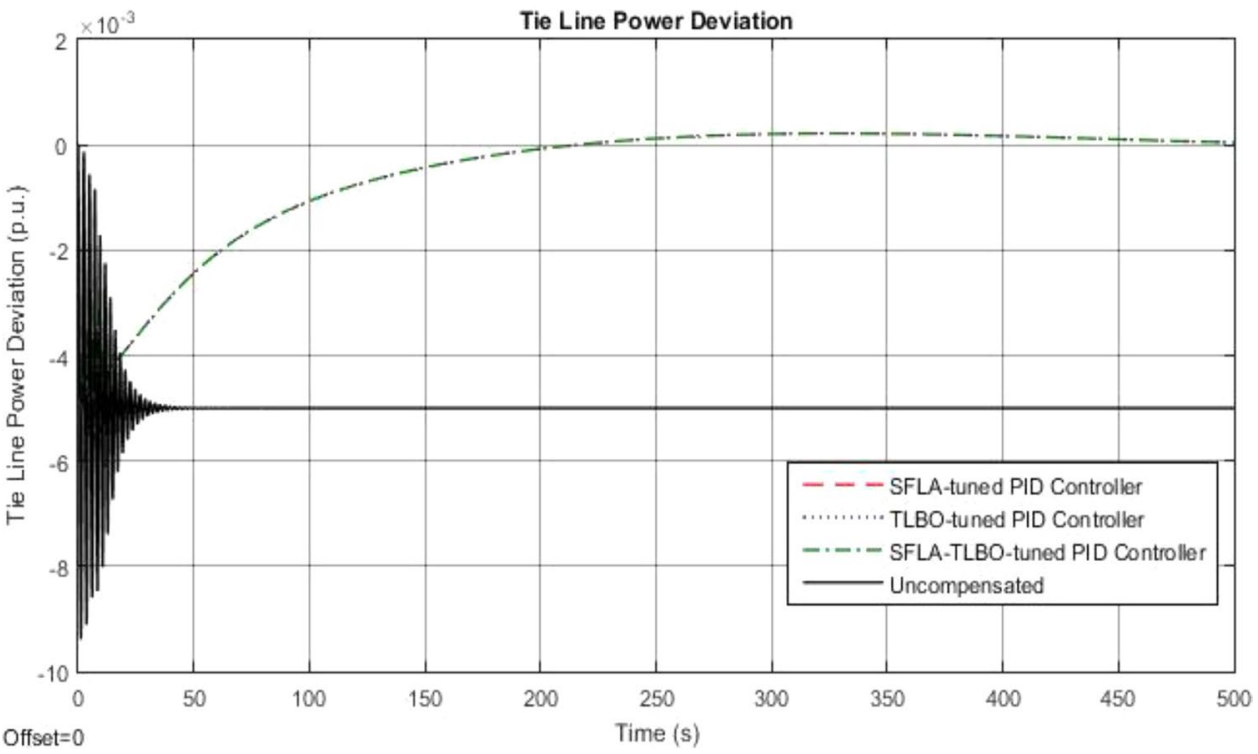


Code availability All codes were implemented on Matlab/Simulink and are available upon request.

\section{Declarations}

Conflict of interest On behalf of all authors, the corresponding author states that there is no conflict of interest.

Consent for publication On behalf of all authors, the corresponding author states that all authors have consented to publish this work in the SN journal of Applied Sciences.

Open Access This article is licensed under a Creative Commons Attribution 4.0 International License, which permits use, sharing, adaptation, distribution and reproduction in any medium or format, as long as you give appropriate credit to the original author(s) and the source, provide a link to the Creative Commons licence, and indicate if changes were made. The images or other third party material in this article are included in the article's Creative Commons licence, unless indicated otherwise in a credit line to the material. If material is not included in the article's Creative Commons licence and your intended use is not permitted by statutory regulation or exceeds the permitted use, you will need to obtain permission directly from the copyright holder. To view a copy of this licence, visit http://creativecommons. org/licenses/by/4.0/.

\section{Appendix}

The parameters for the two-area system are given below.

\begin{tabular}{|c|c|c|c|}
\hline Parameters & Values & Parameters & Values \\
\hline$P_{r 1}$ & $2000 \mathrm{MW}$ & $P_{r 2}$ & $2000 \mathrm{MW}$ \\
\hline$f_{1}$ & $50 \mathrm{~Hz}$ & $f_{2}$ & $50 \mathrm{~Hz}$ \\
\hline $\mathrm{H}$ & 0.0833 & $D$ & 0.00833 \\
\hline $\mathrm{R}$ & $2.4 \mathrm{~Hz} / \mathrm{p} . \mathrm{u}$ & $T_{\text {th }}$ & $1 \mathrm{~s}$ \\
\hline$T_{t}$ & $0.3 \mathrm{~s}$ & $\begin{array}{c}T_{1} \text { (Hydraulic } \\
\text { Governor) }\end{array}$ & $48.7 \mathrm{~s}$ \\
\hline$T_{g}$ & $0.08 \mathrm{~s}$ & $\begin{array}{c}T_{2} \text { (Hydraulic } \\
\text { Governor) }\end{array}$ & $5 \mathrm{~s}$ \\
\hline$T_{\mathrm{tr}}$ & $5 \mathrm{~s}$ & $\begin{array}{c}T_{3} \text { (Hydraulic } \\
\text { Governor) }\end{array}$ & $0.513 \mathrm{~s}$ \\
\hline$T_{\mathrm{r}}$ & $10 \mathrm{~s}$ & B & $0.425 \mathrm{~Hz} / \mathrm{p} . \mathrm{u}$ \\
\hline$T_{12}$ & \multicolumn{3}{|c|}{0.0707 p.u./Hz } \\
\hline
\end{tabular}

The parameters for the non-linear two-area system are as follows.

\begin{tabular}{llll}
\hline Parameters & Values & Parameters & Values \\
\hline $\mathrm{H}$ & 0.0833 & $\mathrm{D}$ & 0.00833 \\
$T_{\mathrm{tr}}$ & $5 \mathrm{~s}$ & $T_{12}$ & $0.08674 \mathrm{p} . \mathrm{u} . / \mathrm{Hz}$ \\
$T_{r}$ & $10 \mathrm{~s}$ & $T_{t}$ & $0.3 \mathrm{~s}$ \\
$\mathrm{R}$ & $2.4 \mathrm{~Hz} /$ p.u & $K_{1}$ & 0.85 \\
$\mathrm{~B}$ & $0.425 \mathrm{~Hz} /$ p.u & $K_{2}$ & 0.095 \\
$K_{3}$ & 0.92 & $C_{\mathrm{B}}$ & 200 \\
\hline
\end{tabular}

\begin{tabular}{llll}
\hline Parameters & Values & Parameters & Values \\
\hline$T_{\mathrm{D}}$ & 0 & $T_{\mathrm{F}}$ & $10 \mathrm{~s}$ \\
$K_{\mathrm{IB}}$ & 0.03 & $T_{\mathrm{IB}}$ & $26 \mathrm{~s}$ \\
$T_{\mathrm{RB}}$ & $69 \mathrm{~s}$ & $T_{\mathrm{sgi}}$ & $0.08 \mathrm{~s}$ \\
\hline
\end{tabular}

\section{References}

1. Kundur P (1994) Power System Stability and Control. McGrawHill, New York

2. Saadat H (1999) Power System Analysis. McGraw-Hill, Singapore

3. Bevrani H (2009) Robust power system frequency control, 1st edition 2nd printing. Springer, Berlin

4. Singh O, Nasiruddin I (2016) Hybrid evolutionary algorithm based fuzzy logic controller for automatic generation control of power systems with governor dead band non-linearity. Cogent Engineering

5. Tushir M, Srivastava S, Arya Y (2012) Application of hybrid fuzzy PID controller for three-area power system with generation rate constraint. Int J Energy Technol Policy 8:159

6. Guha D, Roy P, Banerjee S (2016) Oppositional biogeographybased optimisation applied to SMES and TCSC-based load frequency control with generation rate constraints and time delay. Int J Power Energy Convers 7:391

7. Bhanu P, Bhushan C, Sujatha K et al. (2011) Load frequency controller with PI controller considering non-linearities and boiler dynamics. In: International conference on sustainable energy and intelligent systems (SEISCON 2011)

8. Mazinan A, Hosseini A (2010) Application of intelligence-based predictive scheme to load-frequency control in a two-area interconnected power system. Appl Intell 35:457-468

9. Lee H, Park J, Joo Y (2006) Robust load-frequency control for uncertain nonlinear power systems: A fuzzy logic approach. Inf Sci 176:3520-3537

10. RamaSudha K, Vakula V, Shanthi R (2010) PSO based design of robust controller for two area load frequency control with nonlinearities. IJEST 2:1311-1324

11. Bevrani H, Daneshmand P (2012) Fuzzy logic-based load-frequency control concerning high penetration of wind turbines. IEEE Syst J 6:173-180

12. Soundarrajan A, Sumathi S (2009) Effect of non-linearities in fuzzy based load frequency control. Int J Electron Eng Res 1:37-51

13. Kazemi M, Karrari M, Menhaj M (2003) Decentralized robust adaptive load frequency control using interactions estimation. Elect Eng (Archiv fur Elektrotechnik) 85:219-227

14. Kunya A, Argin M, Kucuksari S (2019) Optimal load frequency control of multi-area power system considering incremental control action. In: 2019 IEEE texas power and energy conference (TPEC)

15. Huang C, Zheng Q (2014) Application of linear active disturbance rejection control to power system load frequency control. Int J Intell Control Syst 19:1-7

16. Oozeer MY, Ramjug-Ballgobin R (2018) Optimization of load frequency control for non-linear system. In: International conference on emerging trends in electrical, electronic and communications engineering. Springer, Cham, pp 11-21

17. Oozeer MY, Ramjug-Ballgobin R (2018) Optimization of load frequency control for non-linear System. In: International conference on emerging trends in electrical, electronic and communications engineering. Springer, Cham, pp 34-44 
18. Guha D, Roy P, Banerjee S (2016) Load frequency control of large scale power system using quasi-oppositional grey wolf optimization algorithm. Eng Sci Technol Int J 19:1693-1713

19. Ahmed J (2014) Optimal load frequency control in a single area power system based Genetic Algorithm. Int J Sci Eng Res 5:2196-2200

20. Ramjug-Ballgobin R, Fowdar R (2019) Application of metaheuristic control strategies to voltage regulation. SN Appl Sci 1(12):1-8

21. Masiala M, Ghribi M, Kaddouri A (2012) A two-layered load and frequency controller of a power system. Fuzzy ControllersRecent Advances in Theory and Applications. IntechOpen

22. Arya Y, Kumar N, Sinha S (2012) Fuzzy logic based load frequency control of multi- area electrical power system considering nonlinearities and boiler dynamics. Int Energy J 13:97-112

23. Guha D, Roy P, Banerjee S (2018) Application of backtracking search algorithm in load frequency control of multi-area interconnected power system. Ain Shams Eng J 9:257-276

24. Eusuff M, Lansey K, Pasha F (2006) Shuffled frog-leaping algorithm: a memetic meta-heuristic for discrete optimization. Eng Optim 38:129-154

25. Azizipanah-Abarghooee $R$, Narimani M, Bahmani-Firouzi B, Niknam T (2014) Modified shuffled frog leaping algorithm for multi-objective optimal power flow with FACTS devices. J Intell Fuzzy Syst 26:681-692
26. Luo J, Li X, Chen M, Liu H (2015) A novel hybrid shuffled frog leaping algorithm for vehicle routing problem with time windows. Inf Sci 316:266-292

27. Bhattacharjee K, Sarmah S (2014) Shuffled frog leaping algorithm and its application to 0/1 knapsack problem. Appl Soft Comput 19:252-263

28. Priyambada S, Mohanty P, Sahu B (2014) Automatic voltage regulator using TLBO algorithm optimized PID controller. In: 2014 9th international conference on industrial and information systems (ICIIS)

29. Wang L, Zou F, Hei X et al (2014) A hybridization of teachinglearning-based optimization and differential evolution for chaotic time series prediction. Neural Comput Appl 25:1407-1422

30. Xu Y, Wang L, Wang S, Liu M (2015) An effective teaching-learning-based optimization algorithm for the flexible job-shop scheduling problem with fuzzy processing time. Neurocomputing 148:260-268

31. Rao R, Savsani V, Vakharia D (2011) Teaching-learning-based optimization: A novel method for constrained mechanical design optimization problems. Comput Aided Des 43:303-315

Publisher's Note Springer Nature remains neutral with regard to jurisdictional claims in published maps and institutional affiliations. 